\title{
Review
}

\section{Uric Acid Elevation by Favipiravir, an Antiviral Drug}

\author{
Eikan Mishima, ${ }^{1}$ Naohiko Anzai, ${ }^{2}$ Mariko Miyazaki ${ }^{1}$ and Takaaki Abe ${ }^{1,3,4}$ \\ ${ }^{1}$ Division of Nephrology, Endocrinology and Vascular Medicine, Tohoku University Graduate School of Medicine, \\ Sendai, Miyagi, Japan \\ ${ }^{2}$ Department of Pharmacology, Chiba University Graduate School of Medicine, Chiba, Chiba, Japan \\ ${ }^{3}$ Division of Medical Science, Tohoku University Graduate School of Biomedical Engineering, Sendai, Miyagi, \\ Japan \\ ${ }^{4}$ Department of Clinical Biology and Hormonal Regulation, Tohoku University Graduate School of Medicine, \\ Sendai, Miyagi, Japan
}

\begin{abstract}
In light of the recent pandemic, favipiravir $\left(\right.$ Avigan $\left.^{\circledR}\right)$, a purine nucleic acid analog and antiviral agent approved for use in influenza in Japan, is being studied for the treatment of coronavirus disease 2019 (COVID-19). Increase in blood uric acid level is a frequent side effect of favipiravir. Here, we discussed the mechanism of blood uric acid elevation during favipiravir treatment. Favipiravir is metabolized to an inactive metabolite M1 by aldehyde oxidase and xanthine oxidase, and excreted into urine. In the kidney, uric acid handling is regulated by the balance of reabsorption and tubular secretion in the proximal tubules. Favipiravir and M1 act as moderate inhibitors of organic anion transporter 1 and 3 (OAT1 and OAT3), which are involved in uric acid excretion in the kidney. In addition, M1 enhances uric acid reuptake via urate transporter 1 (URAT1) in the renal proximal tubules. Thus, favipiravir is thought to decrease uric acid excretion into urine, resulting in elevation of uric acid levels in blood. Elevated uric acid levels were returned to normal after discontinuation of favipiravir, and favipiravir is not used for long periods of time for the treatment of viral infection. Thus, the effect on blood uric acid levels was subclinical in most studies. Nevertheless, the adverse effect of favipiravir might be clinically important in patients with a history of gout, hyperuricemia, kidney function impairment (in which blood concentration of M1 increases), and where there is concomitant use of other drugs affecting blood uric acid elevation.
\end{abstract}

Keywords: COVID-19; favipiravir; hyperuricemia; influenza; SARS-CoV-2

Tohoku J. Exp. Med., 2020 June, 251 (2), 87-90.

\section{Introduction}

Favipiravir $\left(\operatorname{Avigan}^{\circledR}\right)$ is an antiviral agent that inhibits the RNA-dependent RNA polymerase of many RNA viruses (Furuta et al. 2002, 2017). In Japan, favipiravir has been approved for treating influenza strains unresponsive to current antivirals. Given the wide anti-viral spectrum of favipiravir, it is also being studied in the context of the recent pandemic of coronavirus disease 2019 (COVID-19) (Du and Chen 2020), which is caused by infection with severe acute respiratory syndrome coronavirus 2 (SARS-CoV-2). Increase in blood uric acid level is the most frequent side effect of favipiravir (PMDA, Pharmaceuticals and Medical Devices Agency 2014; Fujifilm, Fujifilm Toyama Chemical
Co., Ltd. 2019). Uric acid is a product of the metabolic breakdown of purine nucleotides. Hyperuricemia, which is an abnormally high uric acid level in the blood, can lead to gout and is associated with other medical conditions including kidney function impairment. This review summarizes the mechanism for blood uric acid elevation caused by favipiravir.

\section{Metabolism of Favipiravir}

Favipiravir is a purine nucleic acid analog and pyrazinecarboxamide derivative. It is a prodrug that is metabolized to its active form, favipiravir-ribofuranosyl-5'-triphosphate (favipiravir-RTP) in cells (Furuta et al. 2005). Human hypoxanthine guanine phosphoribosyltransferase

Received May 11, 2020; revised and accepted May 29, 2020. Published online June 12, 2020; doi: 10.1620/tjem.251.87.

Correspondence: Eikan Mishima, M.D., Ph.D., Division of Nephrology, Endocrinology and Vascular Medicine, Tohoku University

Graduate School of Medicine, 1-1 Seiryo-machi, Aoba-ku, Sendai, Miyagi 980-8574, Japan.

e-mail: eikan@med.tohoku.ac.jp

(C)2020 Tohoku University Medical Press. This is an open-access article distributed under the terms of the Creative Commons Attribution-NonCommercial-NoDerivatives 4.0 International License (CC-BY-NC-ND 4.0). Anyone may download, reuse, copy, reprint, or distribute the article without modifications or adaptations for non-profit purposes if they cite the original authors and source properly.

https://creativecommons.org/licenses/by-nc-nd/4.0/ 
(HGPRT), an enzyme in the purine salvage pathway (Hirano and Peters 2016; Mishima et al. 2020), was reported to play a key role in this activation process (Naesens et al. 2013). Favipiravir-RTP competes with purine nucleosides and interferes with viral replication by incorporation into the virus RNA, thus potentially inhibiting the RNA-dependent RNA polymerase of RNA viruses (Furuta et al. 2005) (Fig. 1A). Favipiravir undergoes metabolism in the liver mainly by aldehyde oxidase and partially by xanthine oxidase, producing an inactive metabolite favipiravir-M1 excreted by the kidneys (Madelain et al. 2016). Favipiravir is extensively metabolized with only $1 \%$ recovered unchanged in urine. Cytochrome P450 isoenzymes do not contribute to the metabolism of favipiravir (PMDA 2014; Fujifilm 2019).

\section{Effect of Favipiravir on Uric Acid Levels}

Favipiravir can increase blood uric acid levels. In phase I-II safety studies, the effect was observed in a dosedependent manner (PMDA 2014; Fujifilm 2019). In a study of healthy volunteers, favipiravir increased blood uric acid in 7 out of 8 volunteers $(87.5 \%)$. Blood uric acid levels were elevated by an average of $4.4 \mathrm{mg} / \mathrm{dL}$ over baseline after 6 days of favipiravir administration $(3,200 \mathrm{mg}$ on day 1 followed by $1,200 \mathrm{mg}$ on days 2-5) and returned to normal 7 days after discontinuation (MHLW, Ministry of Health, Labour and Welfare 2016). In a phase III clinical study that compared the treatment efficacy of favipiravir to oseltamivir for patients with influenza, the incidence of elevated blood uric acid levels in the favipiravir group was higher $(5.6 \%, 21 / 378)$ than that in an oseltamivir group (PMDA 2014). In the same study and the pharmacokinetic study (PMDA 2014), among 393 subjects administered favipiravir, blood uric acid levels were increased after 3-4 days of administration (1,600 $\mathrm{mg}$ on day 1 followed by 800 $\mathrm{mg}$ on days 2-5). The average elevation was $0.8 \mathrm{mg} / \mathrm{dL}$ in men (baseline $5.6 \mathrm{mg} / \mathrm{dL}$ ) and $1.4 \mathrm{mg} / \mathrm{dL}$ in women (baseline $3.9 \mathrm{mg} / \mathrm{dL}$ ). The blood uric acid levels were returned to baseline level upon cessation of treatment. There has been no evidence that hyperuricemia caused by favipiravir leads to clinical manifestations; however, these studies excluded individuals with a history of gout and those taking medication for hyperuricemia. In a randomized control trial involving patients with COVID-19 to compare the efficacy and safety of favipiravir and umifenovir (Arbidol ${ }^{\circledR}$ ), which is an antiviral drug for influenza infection used in Russia and China (Chen et al. 2020), the incidence of elevated blood uric acid was significantly higher in the favipiravir group (16/116 patients, 14\%) than in the umifenovir group (3/120 patients, $2.5 \%$ ). In an interim report of a multicenter observational study of favipiravir in COVID-19 patients in Japan (Fujita Health University Hospital 2020), 15.5\% $(335 / 2158)$ of the patients showed hyperuricemia or elevation of blood uric acid level by favipiravir treatment, whose dose was 3,600 $\mathrm{mg}$ on day 1 followed by $1,600 \mathrm{mg}$ on the subsequent days (median administration period, 11 days). In addition, 8 patients $(0.37 \%)$ presented gout. Thus, favipiravir can also increase blood uric acid levels in COVID-19 patients; however, these studies did not show data about the detailed uric acid levels.

\section{Mechanism of Uric Acid Elevation by Favipiravir}

The elevation of blood uric acid by favipiravir is thought to be the result of decreased excretion of uric acid into the urine (PMDA 2014; Fujifilm 2019). Indeed, among healthy subjects administered favipiravir, urine uric acid levels were decreased in four subjects (1.7\%: 4/242), and blood uric acid levels were increased in these four subjects,
A

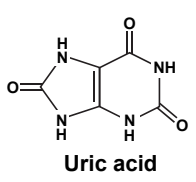

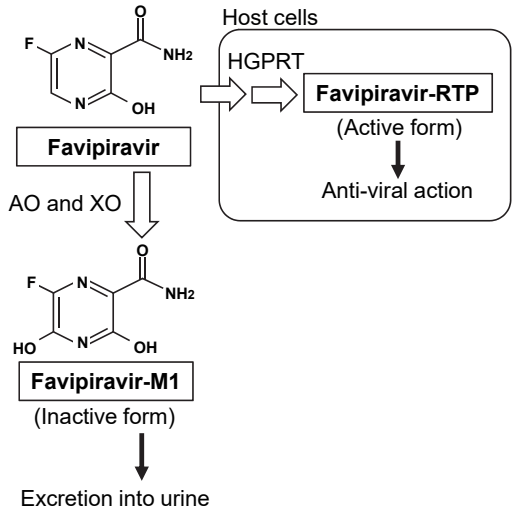

B

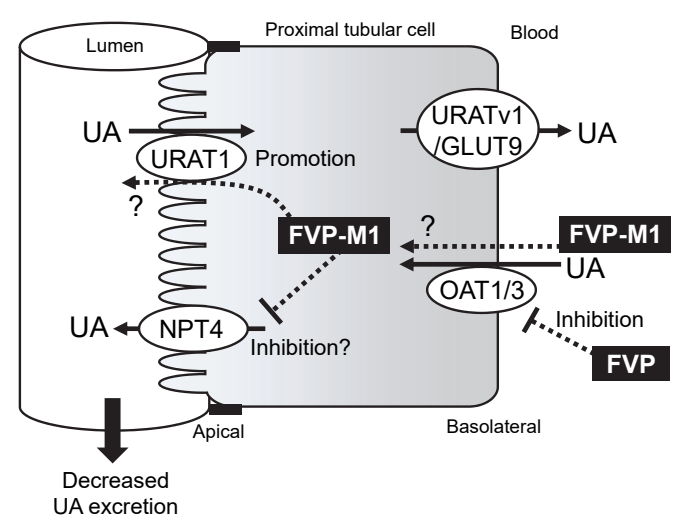

Fig. 1. Favipiravir elevates blood uric acid levels by decreasing urinary excretion.

A. Metabolism and structure of favipiravir.

B. Putative mechanism of uric acid elevation by favipiravir (FVP).

AO, aldehyde oxidase; XO, xanthine oxidase; HGPRT, hypoxanthine guanine phosphoribosyltransferase; RTP, ribofuranosyl-5'-triphosphate; UA, uric acid, URAT1, urate transporter 1; URATv1, voltage-driven urate efflux transporter; GLUT9, glucose transporter 9; OAT 1 and 3, organic anion transporter 1 and 3; NPT4, sodium-dependent phosphate transport protein 4 . 
suggesting that the elevation of blood uric acid level was attributed to the decreased urinary excretion of uric acid by the drug (PMDA 2014). In the kidney, urate handling is regulated by the balance of reabsorption and tubular secretion in the proximal tubules (Fig. 1B). Urate transporter 1 (URAT1) ensures the reabsorption of uric acid on the apical membrane, and organic anion transporter 1 and 3 (OAT1 and OAT3) transport urate for luminal excretion on the basolateral side (Otani et al. 2017; Misawa et al. 2020). Favipiravir and the inactive metabolite M1 are moderate inhibitors of OAT1 and OAT3. In an experiment with cultured cells expressing human transporters (OAT1, OAT3, and URAT1), $800 \mu \mathrm{mol} / \mathrm{L}(126 \mu \mathrm{g} / \mathrm{mL})$ favipiravir inhibited the transporter-mediated uptake of standard substrates. The uptake activity of OAT1, OAT3, and URAT1 was decreased to $30.9 \%, 50.0 \%$, and $65.7 \%$ of the control, respectively (PMDA 2014; Fujifilm 2019). M1 also inhibited the uptake of standard substrates by OAT1, OAT3, and URAT1 at 300 $\mu \mathrm{mol} / \mathrm{L}(51.9 \mu \mathrm{g} / \mathrm{mL})$, decreasing their uptake activity to $45.4 \%, 57.7 \%$, and $31.0 \%$ of the control, respectively. In contrast, M1 enhanced URAT1-mediated urate uptake in a concentration-dependent manner. Thus, decreased tubular secretion of uric acid by inhibition of OAT 1 and OAT3 and promotion of uric acid reuptake in tubules via URAT1 are believed to be the mechanism of blood uric acid elevation by favipiravir (Fig. 1B). In this mechanism, we hypothesize that i) M1 may be a substrate of OAT1, OAT3, and URAT1, and ii) M1 is transported from the blood into the renal proximal tubular cells via OAT1 and OAT3, and then M1 is excreted into the tubular lumen via URAT1 as a counter-substrate of urate, resulting in its trans-stimulatory effect on URAT1 (Fig. 1B). In addition, M1 might inhibit sodium-dependent phosphate transport protein 4 (NPT4)dependent urate secretion in the apical side (Jutabha et al. 2011). An experiment in cultured cells expressing human transporters showed that favipiravir was not a substrate of human organic anion transporters (OAT1, OAT2, and OAT3), organic cation transporters (OCT1, OCT2, and OCT3), organic anion transport polypeptide (OATP2), URAT1, and P-glycoprotein (PMDA 2014; Fujifilm 2019). However, it remains unknown whether M1 is a substrate of these transporters.

The approved favipiravir regimen for influenza in Japan, which includes a 3,200 mg oral loading dose $(1,600$ mg every 12 hours) on day 1 followed by $600 \mathrm{mg}$ twice daily on days $2-5$, maintains plasma concentration of the drug $>20 \mu \mathrm{g} / \mathrm{mL}$ during the administration period and reached a maximal concentration of $60 \mu \mathrm{g} / \mathrm{mL}$ (PMDA 2014; Fujifilm 2019). Thus, the concentration of the drug that influenced the transporters' activities in the in vitro studies is nearly comparable to the blood concentration of the drug in the individuals receiving favipiravir. Although favipiravir is partially metabolized by xanthine oxidase, an in vitro study indicated that favipiravir does not influence xanthine oxidase activity (PMDA 2014; Fujifilm 2019). The effect of favipiravir on the activity and expression of
HGPRT has not been examined.

\section{Drug Interaction Effects on Uric Acid Levels}

Other agents that elevate uric acid levels can interact with favipiravir. Pyrazinamide, an antituberculous drug, increases serum uric acid levels (Louthrenoo et al. 2015). A metabolite of pyrazinamide, pyrazinoic acid, enhances urate reuptake by URAT1, resulting in decreased uric acid excretion into the urine (Ben Salem et al. 2017). In healthy subjects, pyrazinamide elevated the blood uric acid level from 6.2 to $11.6 \mathrm{mg} / \mathrm{dL}$, and concomitant favipiravir administration further elevated the level to $13.9 \mathrm{mg} / \mathrm{dL}$ (PMDA 2014). Thus, concomitant use of other urate elevating drugs may enhance the uric acid elevation caused by favipiravir.

When favipiravir is administered concomitantly with an aldehyde oxidase inhibitor, such as raloxifene (Obach et al. 2004), or a xanthine oxidase inhibitor, such as allopurinol, the metabolic clearance of favipiravir may decrease, resulting in increased plasma concentrations of favipiravir and decreased concentration of M1. However, no study has examined the effect on the plasma concentration of favipiravir by a concomitant use of an aldehyde oxidase inhibitor or xanthine oxidase inhibitor. In phase I/II study, a healthy volunteer with low aldehyde oxidase activity showed remarkably high favipiravir concentration and low M1 concentration in the plasma (PMDA 2014; Fujifilm 2019). However, in the phase III study, the incidence rate of clinical adverse effects was not associated with the aldehyde oxidase activity of the subjects (PMDA 2014; Fujifilm 2019).

\section{Effect in Individuals with Kidney Dysfunction}

In patients with advanced kidney dysfunction (creatinine clearance $<30 \mathrm{~mL} / \mathrm{min}$, not on dialysis), the plasma concentration of favipiravir was reported to be only 1.3 -fold higher than normal (MHLW 2016). In contrast, the plasma concentration of the inactive metabolite M1 was obviously higher because M1 is excreted by the kidney. In a single patient with moderate kidney dysfunction (creatinine clearance $42 \mathrm{~mL} / \mathrm{min}$ ) in the pharmacokinetic study, trough plasma concentrations of M1 were 2.5 times higher than those in patients without renal dysfunction (PMDA 2014; Fujifilm 2019). In addition, patients with kidney dysfunction generally show higher blood uric acid levels and decreased urinary uric acid clearance ( $\mathrm{Li}$ et al. 2018). Thus, in patients with moderate to advanced kidney dysfunction, favipiravir may result in hyperuricemia more frequently due to the exposure to higher plasma concentrations of M1, although direct evidence has not been gathered.

\section{Conclusion}

Favipiravir can elevate blood uric acid levels, but this effect is reversed after discontinuation of the drug. Since favipiravir is not used for long periods of time for the treatment of viral infection, the effect on blood uric acid levels was subclinical in most studies. However, those studies did 
not include subjects with history of gout, hyperuricemia, and/or advanced kidney dysfunction; namely, the side effect on blood uric acid levels has not been fully examined in such populations. Clinicians, therefore, need to be cautious of blood uric acid elevation, when favipiravir is used for patients with a history of gout, hyperuricemia, or kidney dysfunction that can increase blood concentration of M1 and for patients concomitantly treated with other drugs causing blood uric acid elevation. In addition, there are only limited data available as to the suitable treatment of hyperuricemia resulting from favipiravir administration. No study has compared uric acid production inhibitors with uricosuric agents in this situation. To clarify the favipiravir-mediated mechanism of decreased urinary excretion of uric acid, future research should examine whether M1 is a substrate of OAT1, OAT3, and URAT1 and identify the transporters involved in the urinary excretion of M1.

\section{Conflict of Interest}

The authors declare no conflict of interest.

\section{References}

Ben Salem, C., Slim, R., Fathallah, N. \& Hmouda, H. (2017) Drug-induced hyperuricaemia and gout. Rheumatology (Oxford), 56, 679-688.

Chen, C., Zhang, Y., Huang, J., Yin, P., Cheng, Z., Wu, J., Chen, S., Zhang, Y., Chen, B., Lu, M., Luo, Y., Ju, L., Zhang, J. \& Wang, X. (2020) Favipiravir versus arbidol for COVID-19: a randomized clinical trial. medRxiv, doi:10.1101/2020.03.17.20 037432.

Du, Y.X. \& Chen, X.P. (2020) Favipiravir: pharmacokinetics and concerns about clinical trials for $2019-\mathrm{nCoV}$ infection. Clin. Pharmacol. Ther, doi: 10.1002/cpt.1844. [Epub ahead of print].

Fujifilm Toyama Chemical Co., Ltd. (Fujifilm)(2019) The drug interview form of favipiravir (Avigan ${ }^{\mathbb{R}}$ ) (April. 2019 version). http://fftc.fujifilm.co.jp/med/abigan/pack/pdf/abigan_if_01.pdf [Accessed: May 5, 2015] (in Japanese).

Fujita Health University Hospital (2020) Interim report of observational study of favipiravir (May. 26. 2020 version). http://www.kansensho.or.jp/uploads/files/topics/2019ncov/ covid19_favip_0526.pdf [Accessed: May 27, 2015] (in Japanese).

Furuta, Y., Komeno, T. \& Nakamura, T. (2017) Favipiravir (T-705), a broad spectrum inhibitor of viral RNA polymerase. Proc. Jpn. Acad. Ser. B Phys. Biol. Sci., 93, 449-463.

Furuta, Y., Takahashi, K., Fukuda, Y., Kuno, M., Kamiyama, T., Kozaki, K., Nomura, N., Egawa, H., Minami, S., Watanabe, Y., Narita, H. \& Shiraki, K. (2002) In vitro and in vivo activities of anti-influenza virus compound T-705. Antimicrob. Agents Chemother., 46, 977-981.

Furuta, Y., Takahashi, K., Kuno-Maekawa, M., Sangawa, H.,
Uehara, S., Kozaki, K., Nomura, N., Egawa, H. \& Shiraki, K. (2005) Mechanism of action of T-705 against influenza virus. Antimicrob. Agents Chemother., 49, 981-986.

Hirano, M. \& Peters, G.J. (2016) Advances in purine and pyrimidine metabolism in health and diseases. Nucleosides Nucleotides Nucleic Acids, 35, 495-501.

Jutabha, P., Anzai, N., Wempe, M.F., Wakui, S., Endou, H. \& Sakurai, H. (2011) Apical voltage-driven urate efflux transporter NPT4 in renal proximal tubule. Nucleosides Nucleotides Nucleic Acids, 30, 1302-1311.

Li, F., Guo, H., Zou, J., Chen, W., Lu, Y., Zhang, X., Fu, C., Xiao, J. \& Ye, Z. (2018) Urinary excretion of uric acid is negatively associated with albuminuria in patients with chronic kidney disease: a cross-sectional study. BMC Nephrol., 19, 95.

Louthrenoo, W., Hongsongkiat, S., Kasitanon, N., Wangkaew, S. \& Jatuworapruk, K. (2015) Effect of antituberculous drugs on serum uric acid and urine uric acid excretion. J. Clin. Rheumatol., 21, 346-348.

Madelain, V., Nguyen, T.H., Olivo, A., de Lamballerie, X., Guedj, J., Taburet, A.M. \& Mentre, F. (2016) Ebola virus infection: review of the pharmacokinetic and pharmacodynamic properties of drugs considered for testing in human efficacy trials. Clin. Pharmacokinet., 55, 907-923.

Ministry of Health, Labour and Welfare (MHLW) (2016) Company data about Avigan ${ }^{\circledR}$ in Pandemic Influenza Subcommittee on November, 25, 2016.

https://www.mhlw.go.jp/file/05-Shingikai-10601000-Daijink anboukouseikagakuka-Kouseikagakuka/0000143922.pdf [Accessed: May 5, 2015] (in Japanese).

Misawa, K., Hasegawa, T., Mishima, E., Jutabha, P., Ouchi, M., Kojima, K., Kawai, Y., Matsuo, M., Anzai, N. \& Nagasaki, M. (2020) Contribution of rare variants of the SLC22A12 gene to the missing heritability of serum urate Levels. Genetics, 214, 1079-1090.

Mishima, E., Mori, T., Nakajima, Y., Toyohara, T., Kikuchi, K., Oikawa, Y., Matsuhashi, T., Maeda, Y., Suzuki, T., Kudo, M., Ito, S., Sohara, E., Uchida, S. \& Abe, T. (2020) HPRT-related hyperuricemia with a novel p.V35M mutation in HPRT1 presenting familial juvenile gout. CEN Case Rep., doi: 10.1007/s13730-020-00459-9. [Epub ahead of print].

Naesens, L., Guddat, L.W., Keough, D.T., van Kuilenburg, A.B., Meijer, J., Vande Voorde, J. \& Balzarini, J. (2013) Role of human hypoxanthine guanine phosphoribosyltransferase in activation of the antiviral agent T-705 (favipiravir). Mol. Pharmacol., 84, 615-629.

Obach, R.S., Huynh, P., Allen, M.C. \& Beedham, C. (2004) Human liver aldehyde oxidase: inhibition by 239 drugs. $J$. Clin. Pharmacol., 44, 7-19.

Otani, N., Ouchi, M., Hayashi, K., Jutabha, P. \& Anzai, N. (2017) Roles of organic anion transporters (OATs) in renal proximal tubules and their localization. Anat. Sci. Int., 92, 200-206.

Pharmaceuticals and Medical Devices Agency (PMDA) (2014) Report on the deliberation results: avigan. Japan; Evaluation and Licensing Division, Pharmaceutical and Food Safety Bureau. http://www.pmda.go.jp/files/000210319.pdf [Accessed: May 5, 2020]. 